\title{
Quantitative assessment of defect characterisation using TSR coefficient images
}

\author{
by Daniel L. BALAGEAS*, Jean-Michel ROCHE**, François-Henry Leroy** \\ *Univ. Bordeaux, I2M, TREFLE Dept., UMR 5295, F-33400 Talence, France, daniel.balageas@wanadoo.fr \\ ** ONERA, Composite Materials and Structures Dept., BP72, 92322 Châtillon cedex, France
}

\begin{abstract}
It has been recently proposed to use the TSR coefficient images to detect defects in structures. The ability of this approach has been qualitatively achieved and compared to well-established processing methods: PPT, PCA and HOS. Based on the highest number of detected defects, the TSR-coefficient images appeared to be at least as good as or even better than the images produced by any of the other techniques. No matter how definite this result is, it still can be objected that a more thorough comparison, based on the assessment of several unbiased parameters, is needed. To address and overcome this lack, the present paper carries on the comparative study over the following metrics: the contrast-to-noise ratio, the sharpness of the defect edges and the accuracy of the identified characteristic dimension of the defect using the contrast full-width at half-maximum (FWHM). Once properly defined, these parameters are assessed for the TSR coefficient profiles in the defective zones, as well as for the PPT, PCA and HOS images. Final, quantitative conclusions on the most appropriate method are then deduced and discussed.
\end{abstract}

\section{Introduction}

It has been recently shown that the images of the logarithmic polynomial coefficients, used to fit the pulseheating thermograms and reduce their noise, according to the Thermographic Signal Reconstruction (TSR) technique [14], could be directly used to provide a unique all-defect-imaging map [5]. The ability of this approach to detect defects has been compared to well-established methods: PPT (pulse phase thermography), PCA (principal component analysis) and HOS (high order statistics). Based on the highest number of detected defects in a coupon with well-known artificial embedded defects, the TSR method appeared at least as good as or even better than any of the other techniques. No matter how definite this result is, it still can be objected that a more thorough comparison, based on the assessment of several unbiased parameters, is needed: this is the aim of the present work.

After a brief recall of the TSR technique, and the description of the experimental data that will be exploited, the methodology followed for the quantification of the "quality" of the defect detection and imaging is presented. It is based on the definition of three metrics: the contrast-to-noise ratio (CNR), the sharpness of the defect edges and the accuracy of the identified characteristic dimension of the defect using the contrast full-width at half-maximum (FWHM). These parameters are first assessed for the TSR coefficient profiles in the defective zones, and then for the PPT, PCA and HOS images. Final, quantitative conclusions on the most appropriate method are then deduced and discussed

\section{Recall of the TSR coefficient images method}

The TSR method initially developed for pulse thermography [1-4] and recently for step-heating $[6,7]$ consists in:

- The fitting of the experimental log-log plot thermogram by a logarithmic polynomial of degree $n$ :

$$
\ln _{10}(\Delta T)=a_{0}+a_{1} \ln _{10}(t)+a_{2}\left[\ln _{10}(t)\right]^{2}+\ldots+a_{n}\left[\ln _{10}(t)\right]^{n}
$$

with $\Delta T$ the temperature increase as a function of time $\mathrm{t}$ (thermogram) for each pixel (i,j). This fitting replaces the full sequence of temperature rise images, $\Delta T(\mathrm{i}, \mathrm{j}, t)$ by the series of $(\mathrm{n}+1)$ images of the polynomial coefficients: $a_{0}(\mathrm{i}, \mathrm{j}), \ldots$ $a_{n}(\mathrm{i}, \mathrm{j})$, from which a full thermographic sequence can be rebuilt.

- The computation of the $1^{\text {st }}$ and $2^{\text {nd }}$ logarithmic derivatives of the thermograms, the derivation being achieved directly on the polynomial, with a limited increase of the temporal noise.

The sequences of images of the derivatives can be either qualitatively observed to detect defects (Figure 1), or quantitatively used to evaluate the depth of defects from characteristic times. The so-obtained defect images may have better signal-to noise ratio (SNR) and sharpness than the crude thermographic images, but it is necessary to select several images, each one being adapted to a specific depth range in relation with the possibly existing defects. This is the "classic" use of the TSR method. To lead to precise derivatives, the degree of the fitting polynomial must be high. For instance, it was empirically found that $n=11$ was a satisfying degree for delamination-like defects [5].

It has been recently proposed to use directly the images of the polynomial coefficients to detect the defects. Even better, it was shown how to build a unique "composite" image of the defects (see Figure 1) by projecting a trio of coefficient images in an RGB basis [5]. For delamination-like defects, the best detection was obtained for the polynomial degree $n=7$, which happens to be lower than the one required for a proper use of the derivative images. 


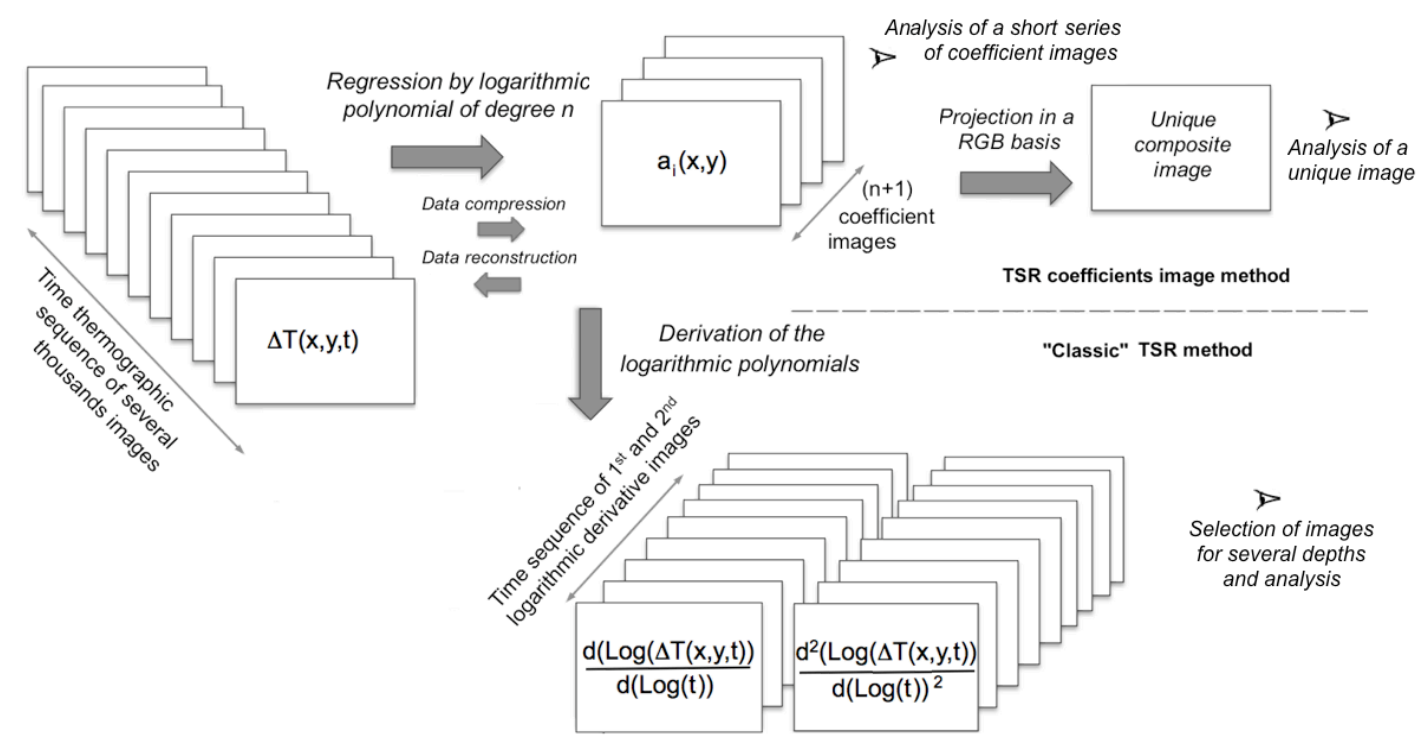

Figure 1. Defect detection by the TSR method.

\section{Analysed data}

The experimental data previously used for a qualitative study [5] is considered again. It results from the recording of the time-evolution of the front-face temperature of a $270 \times 200 \times 5.25 \mathrm{~mm}^{3}$ carbon/epoxy composite plate, already studied in the past [8,9]. The coupon, coated on both faces by a black, thin, carbon layer, contained $80 \mu \mathrm{m}$-thick Teflon $^{\circledR}$ inserts of various sizes and depths (see Figure 2).

The pulse thermography test was performed with two Elinchrom flash lamps delivering a pulse of total energy $6 \mathrm{~kJ}$ in $4 \mathrm{~ms}$. Temperature images of $320 \times 256$ pixels were recorded during $60 \mathrm{~s}$ at a frequency of $200 \mathrm{images} / \mathrm{s}$, with an integration time of $230 \mu \mathrm{s}$, by a CEDIP Jade LWIR camera. Other tests have been performed more recently on the same coupon, with a FLIR X6540sc infrared camera, with a better spatial resolution $(640 \times 512$ pixels) and fewer defective pixels ("dead" or erratic) [10]; however, this paper is based on the first set of data since it has been more extensively studied and already processed with the previously mentioned techniques (TSR, PCT, PPT and HOS) [5]. Consequently, the metrics here evaluated, which depends on both the performance of the thermographic acquisition system and the data processing methods, are not representative of the best of the present state-of-the art and have only a relative value, nonetheless useful and relevant for the assessment and comparison of these data processing techniques.

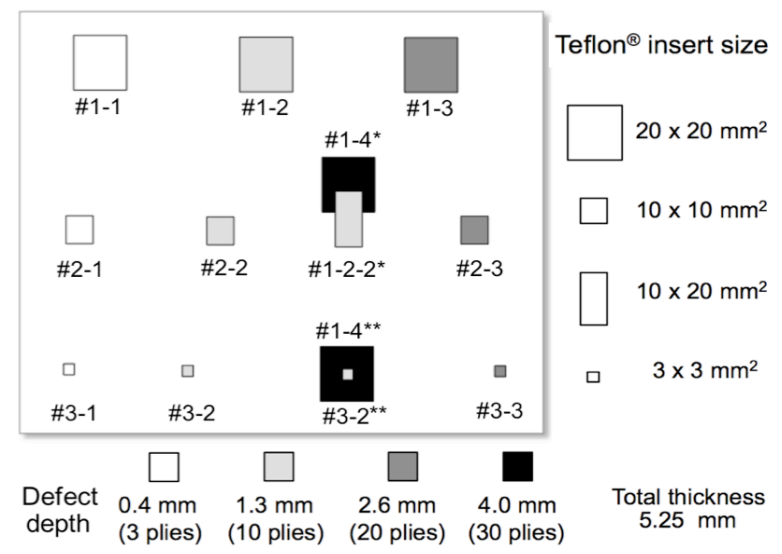

Figure 2. Tested carbon/epoxy coupon with its artificial defects.

\section{Methodology of evaluation of the parameters characterising the defect images: contrast-to-noise ratio, sharpness and lateral dimension}

To quantitatively assess the defect detection based on the coefficient images, several parameters need to be identified. These parameters are different depending on whether the grey-tone polynomial coefficient images or the RGB colour composite image are considered. In this study, the grey-tone images of the polynomial coefficients are only considered. The studied parameters are: 
- the signal-to-noise ratio (SNR) of the contrast, which will be referred as the contrast-to-noise ratio (CNR) from now on,

- the sharpness of the image,

- the characteristic dimension of the defect image compared to the actual one.

The first difference between the present work and previous quantitative comparisons of data processing for pulse thermography lies in the fact that the assessment of the defect signatures is usually based on time-dependent parameters, such as temperature rise, contrast, or logarithmic derivatives, which makes it necessary to analyse their time evolution [4] or to define the proper observation times, for instance when maximum contrasts occur [11]. In the present work, the approach radically differs since it is based on the coefficient images, each one of them integrating information linked to the full duration of the experiment.

The second difference lies in the very definition of the CNR. Most authors have considered the mean value of the signal in the defective area [11,12], the limits of which are difficult to evaluate when the bluntness is important. The present approach consists in the analysis of profiles along lines crossing the regions of interest (ROls), i.e. the defect signatures and their surroundings. Figure 3 presents the $a_{5}$ TSR coefficient image with the superimposed grid of rows and columns for which coefficient distribution is explored. The size of the image is $194 \times 166$ pixels and a pixel equals $0.87 \times 0.87 \mathrm{~mm}^{2}$. Hence the nominal defect dimensions, $D_{d}$, of $23 \mathrm{px}, 11.5 \mathrm{px}$ and $3.5 \mathrm{px}$ respectively for the largest inserts $\left(20 \times 20 \mathrm{~mm}^{2}\right)$, the intermediate-sized ones $\left(10 \times 10 \mathrm{~mm}^{2}\right)$ and the smallest ones $\left(3 \times 3 \mathrm{~mm}^{2}\right)$. These characteristics are reported in Table I given further.

The profile along row 85 of the TSR coefficient of rank 5 of polynomials of degree $7\left(a_{5 / 7}\right)$ is given on Figure 4 as an example, highlighting the ROIs corresponding to the defects of medium size. A few pixels presenting isolated erratic amplitudes can be spotted. They are due to the low quality of the used camera. For the analysis of the coefficient profiles, such pixels have been replaced by the mean of the two adjacent pixels when presenting a gap higher than three times the standard deviation of the noise. The reason is that the parameters are deduced from the local coefficient profiles and that such deviant pixels induce large, non-physical, errors in their evaluations.

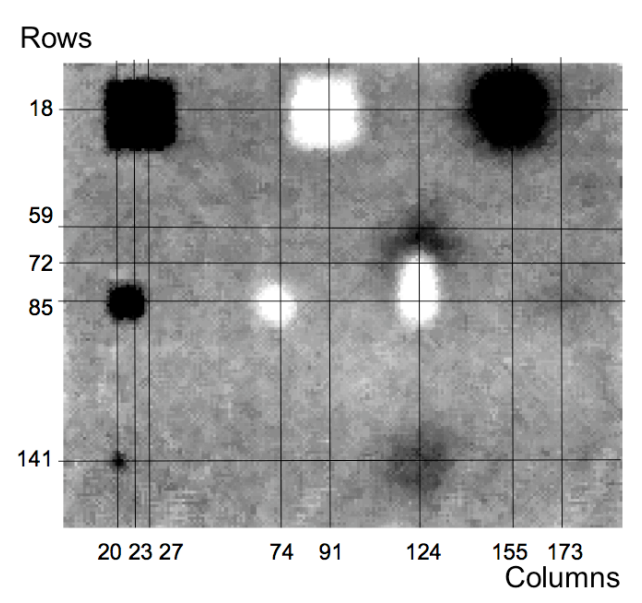

Figure 3. $a_{5 / 7} T S R$ coefficient image with the superimposed grid of rows and columns along which metrics are evaluated.

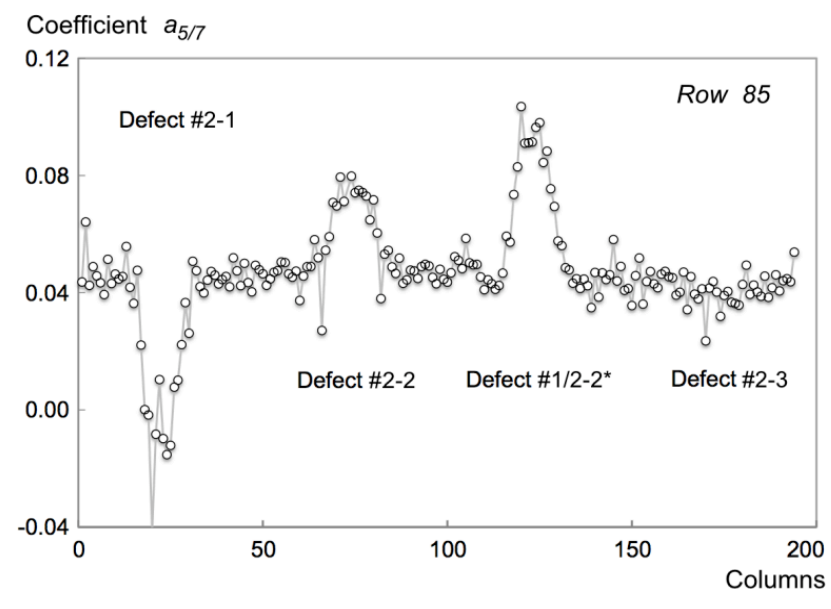

Figure 4. Distribution of the $a_{5 / 7}$ coefficients along row 85.

\subsection{Contrast-to-noise ratio (CNR)}

The CNR may be considered as the ratio of the maximum power contrast $\left(C_{A_{\max }}\right)^{2}$ to the square of the standard deviation $\sigma_{s}^{2}$ of the sound zone in the vicinity of the defects:

$$
\mathrm{CNR}=\left(C_{A_{\max }} / \sigma_{s}\right)^{2},
$$

where $C_{A_{\text {max }}}$ is the maximum of the amplitude contrast between a defect and the sound zone. The CNR can be expressed in decibels as follows:

$$
\text { CNR }=10 \log _{10}\left[\left(C_{A_{\max }} / \sigma_{s}\right)^{2}\right]=20 \log _{10}\left(C_{A_{\max }} / \sigma_{s}\right) \text {. }
$$

In practice, for the search of the maximum contrast of a defective zone, two cases are possible (see Figure 5):

- the defect has a large lateral extent (high aspect ratio $\mathrm{AR}=D_{d} / z_{d}$ ), so that its contrast signature shows a plateau (Figure 5a),

- the defect has a small lateral extent (low aspect ratio), so that its contrast signature shows a Gaussian-like shape (Fig. 5b).

In the following figures, the pixels identified as parts of the sound material baseline in the region of the defect, along a line (row or column) getting close to the centre of the defect, are represented by black circles, the defect plateau 
by light grey circles, and the defect edges by white circles. When the plateau does not exist the defect signature is represented by white circles.

The baseline, the plateau and the edges are locally fitted by least-mean square polynomials (generally of degree 1). When no plateau can be defined, a fitting operation is performed, using a degree 2 or higher polynomial. The fitted values obtained for the central zone of the defect are then used to assess either the contrast between the plateau and the baseline, in the case of a high aspect ratio, or the maximum contrast if no plateau is spotted, in the case of a low or medium aspect ratio.

The noise is assimilated to the standard deviation of the points of the baseline, $\sigma_{s}$, allowing the evaluation of the CNR.

(a)

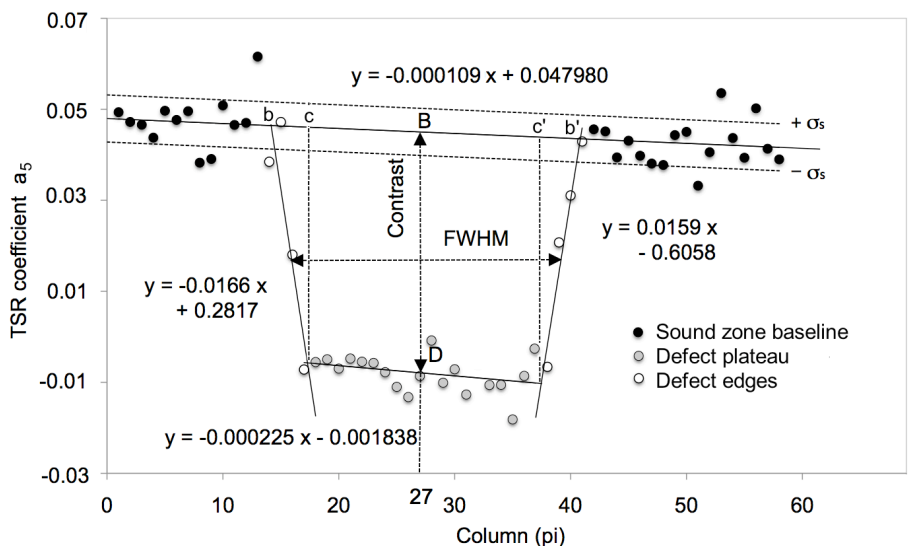

\begin{tabular}{l} 
- Sound material baseline along \\
row 18 \\
$\sigma_{s}=0.0053$ \\
Defect signature \#1-1 \\
Contrast: $\overline{\mathrm{BD}}=-0.053$ \\
$\mathrm{CNR}=-10.1=20.1 \mathrm{~dB}$ \\
Sharpness metrics: \\
$0.5\left(1 / \overline{\mathrm{bc}}+1 / \mathrm{c}^{\prime} \mathrm{b}^{\prime}\right)=0.31 \mathrm{pi}^{-1}$ \\
$\mathrm{FWHM}=23.4 \mathrm{pi}$ \\
\hline
\end{tabular}

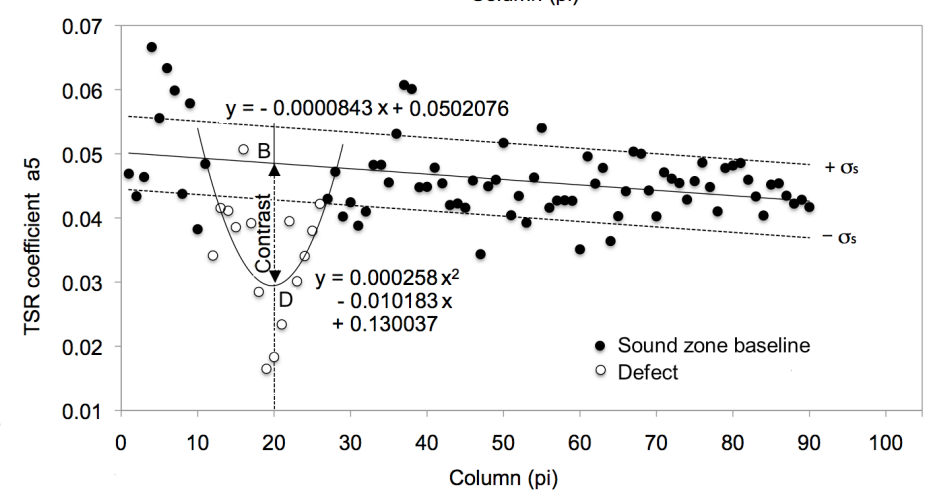

- Sound material baseline along
row 141
$\sigma_{\mathrm{s}}=0.0057$
Defect signature \#3-1
Contrast: $\mathrm{BD}=-0.019$
$\mathrm{CNR}=3.3=10.4 \mathrm{~dB}$

Figure 5. Quantitative characterisation of a defect signature. (a) Defect \#1-1: no strong 3-D effects due to lateral conduction (large aspect ratio defect, $A R=50$ ); (b) Defect \#3-1: 3-D effects due to lateral conduction (medium aspect ratio, AR = 7.5).

\subsection{Sharpness metrics}

Almost all works aiming at quantitatively comparing data processing methods for thermographic NDE are based on the sole CNR parameter. This approach does not take into account the sharpness, which appears to be another relevant parameter regarding the quality of the infrared image. The main reason may be the difficulty to define a metrics to characterize it [12]. A second explanation can be added: most of the comparisons are based on coupons including defects of simple and known shapes, most currently square or circular defects, which helps for the detection and consequently gives less importance to sharpness in the recognition and detection of defects.

Until recently, very few research scientists [4] felt concerned by IR image sharpness, people essentially giving attention to CNR. So, just one measurement of sharpness of IR defect images has been encountered in the literature [12]: the authors chose as sharpness metrics the magnitude of the gradient existing at the edge of the defect, the border between defective and sound zones. The gradients are calculated by finite differences from the pixel intensities of this zone, along orthogonal directions (columns and rows). The sharpness metrics is defined as the square root of the sum of the squares of the $x$ and $y$ gradients. This evaluation is made directly on the thermogram when the contrast is maximum so that both parameters (CNR and sharpness) are calculated at the same time.

In the present work, these evaluations are done for the three coefficients $a_{5 / 7}, a_{6 / 7}$ and $a_{-7 / 7}$ from which the RGB composite image is elaborated (see [5]). The sharpness metrics is calculated from the locally fitted signal, according to the formula given in Figure $5 \mathrm{a}$. The metrics is normalized to facilitate the comparison between the different defect signatures. For that, the gap between the plateau of contrast, when existing, and the sound material baseline is set equal to 1 for each defect, resulting a metrics value between 1 (when there is no pixel between the fitted plateau and the fitted baseline) and $1 /(n+1)$ (when there are $n$ such pixels). An arithmetic mean is calculated for both sides of the plateau for a given line (row or column). In the case of a medium or low aspect ratio, the plateau disappears and this procedure does not work. No identification of the sharpness is made in this case. 


\subsection{Defect lateral extent}

Pulse infrared thermographic images contain defect images of time-dependent dimensions. Defects seem to shrink when time increases, due to edge effects caused by lateral heat diffusion. Almond et al. [13,14] have demonstrated that, in the case of pulse-heating, the lateral dimensions of a defect can be deduced from a linear extrapolation to the origin of time (the pulse occurrence time according to the authors, or better, the emerging contrast time as the present authors recommend), the defect full-width at half-maximum contrast (FWHM) being directly measured on the images as a function of the square root of time. This extrapolation, similar to what is proposed by the early detection approach to identify defect depths [15], mitigates the effects of the lateral diffusion. As pointed out by the authors, the method fails for defects of very low aspect ratios. In fact, it cannot be applied when the plateau disappears (Gaussianshaped contrasts).

In the present case, only one image is to consider, a coefficient image, not a sequence of time-evolving thermogram images. By analogy with Almond's method, the FWHM is measured in the TSR coefficient images. That would be a priori less efficient than the original Almond's method since the coefficient images are influenced by the full thermogram $(\Delta T(t))$, including late information suffering from diffusion effects. Furthermore, this identification is only applied to defect images presenting a plateau, such as defect \#1-1, as illustrated by Figure 5a.

\subsection{Validation of the profile analysis procedure}

To validate the present procedure based on profile analysis in the coefficient images, it is necessary to assess the scatter due to the choice of the analysed profile. That has been done for defect \#1-1 in the $a_{5 / 7}$ image (Table 1). The scatter from various profiles crossing the same defect is assessed for three column profiles $(20,23$ and 27$)$ crossing defect \#1-1 (Figure 3 and Table 1(a)). The scatter is low, around a few percent for contrast $(3.1 \%)$, CNR $(1.8 \%)$ and FWHM (1.7\%). The noise and sharpness metrics are slightly higher ( $7 \%$ and $6 \%$, respectively). This shows that it is possible to carry out such evaluation on a unique profile for a given defect.

Since these profiles can be taken either from a row or a column, the results obtained for column 23 intersecting row 18 and for row 18 intersecting column 23 are compared in Table 1(b). The ratios of the identified parameters are given in the last column: differences lower than $10 \%$ are noticed for contrast, CNR and FWHM. Noise values are a bit higher (13\%) and sharpness values are much higher (55\%). The accuracy on the identified defect dimension using the method presented in section 4.3 is quite remarkable (errors $<5 \%$ ). Such results lead to consider a unique profile for each defect, indifferently from a row or a column. From now on, only rows are analysed.

The order of magnitude of the noise of the sound material zones appears rather uniform on the full sample (standard deviation varying between 0.0030 and 0.0057 ). The CNR appears to depend on both the depth and the characteristic dimension of the defects. The ratio of these two parameters is the governing parameter of the CNR as shown in Figure $6(\mathrm{a})$. For the rectangular defect $(10 \times 20 \mathrm{~mm})$ the characteristic dimension is taken as the square root of the surface. It is important to notice that the curve of Figure 6(a) is specific of the infrared camera used. Indeed, if the contrast is linked to the defect characteristics and mean thermal properties of the material, the noise depends on the performance of the camera and on the heterogeneity of the material/structure.

The sharpness metrics (SM), only to be considered when the contrast presents a plateau, appears correlated to the defect depth as shown in Figure $6(\mathrm{~b})$. The law $\operatorname{SM}\left(1 / z_{d}\right)$ seems to have an asymptote for $z_{d}$ approaching $0.1 \mathrm{~mm}$ : $\mathrm{SM}=0.1 / z_{d}$, SM being evaluated in $\mathrm{pi}^{-1}$ and $z_{d}$ in millimeters.

The accuracy of the identification of the characteristic dimension of the defect, $D_{d}$, taken as the square root of the insert surface, using the FWHM of the contrast when a plateau exists is shown in Figure 6(c) as a function of the reciprocal of the aspect ratio $A R$. The relative errors follow a near linear variation with 1/AR:

$$
\left|\Delta D_{d} / D_{d}\right|_{F W H M} \approx z_{d} / D_{d}
$$

Table 1 - Image analysis of defect \#1-1: (a) discrepancies between column profiles across the defect (analysis following columns), (b) comparison between column and row analysis.

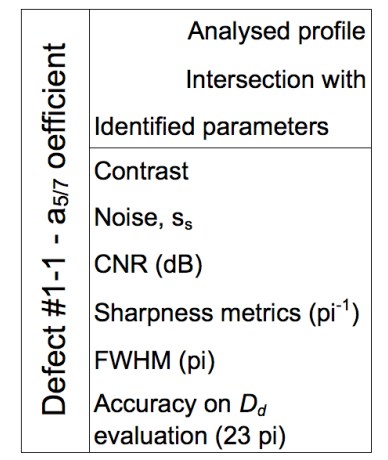

\begin{tabular}{|c|c|c|c|c|}
\hline \multicolumn{3}{|c|}{ Column 20 Column 23 Column 27} & \multirow{2}{*}{$\begin{array}{c}\text { Mean } \\
\text { value } \\
\text { for the } 3 \\
\text { profiles }\end{array}$} & \multirow{2}{*}{$\begin{array}{c}\text { Standard } \\
\text { deviation } \\
\text { for the } 3 \\
\text { profiles }\end{array}$} \\
\hline Row 18 & Row 18 & Row 18 & & \\
\hline$-0,0526$ & $-0,0559$ & $-0,0549$ & $-0,0545$ & 0,0017 \\
\hline 0,0041 & 0,0046 & 0,0042 & 0,0043 & 0,0003 \\
\hline 22,3 & 21,6 & 22,4 & 22,1 & 0,4 \\
\hline 0,16 & 0,17 & 0,15 & 0,16 & 0,01 \\
\hline 22,3 & 22,0 & 22,8 & 22,4 & 0,40 \\
\hline$-2,9 \%$ & $-4,2 \%$ & $-1,0 \%$ & $-2,7 \%$ & $1,6 \%$ \\
\hline
\end{tabular}

(a)

\begin{tabular}{|c|c|c|}
\hline $\begin{array}{c}\text { Column 23 } \\
\text { Row 18 }\end{array}$ & Row 18 & $\begin{array}{c}\text { Ratio of } \\
\text { Cown } 23 \\
\text { colum- } \\
\text { analysis }\end{array}$ \\
\hline$-0,0559$ & $-0,0518$ & 1,08 \\
0,0046 & 0,0044 & 1,05 \\
21,6 & 21,3 & 1,01 \\
0,17 & 0,31 & 0,55 \\
22,0 & 23,4 & 0,94 \\
$-4,2 \%$ & $1,90 \%$ & \\
\hline
\end{tabular}

(b) 


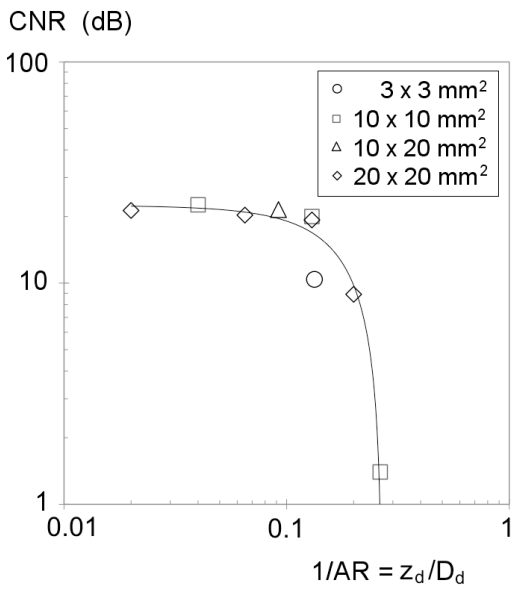

(a)

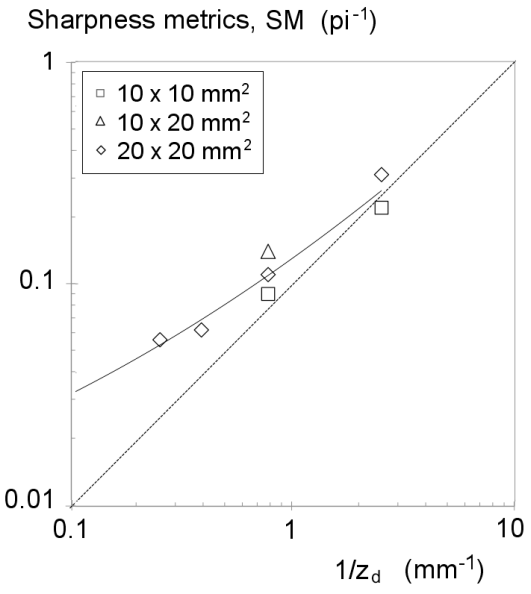

(b)

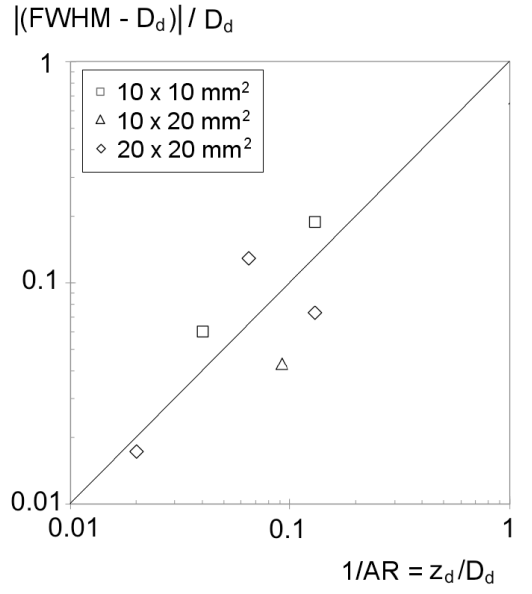

(c)

Figure 6. (a) Influence of the reciprocal of the aspect ratio of defects on the CNR; (b) Influence of the defect depth on the sharpness metrics (considering only the cases leading to plateau-shaped contrasts); (c) Accuracy of the identified defect dimension using the FWHM metrics (considering only the cases leading to plateau-shaped contrasts).

This relation is verified by a factor of \pm 2 . The FWHM for contrasts that do not present a plateau leads to large positive errors. Thus it is not considered. The accuracy of the defect dimension estimate, evaluated as $\log _{10}\left(\left|D_{d} / \Delta D_{d}\right|_{F W H M}\right)$, is the third parameter used for the comparison of images.

\section{Application to the TSR coefficient images and comparison to the images issued from the other three techniques.}

\subsection{Semi-quantitative analysis of the profiles along rows of the images}

Before applying the procedure described above to compare the quality of the images obtained by the TSR coefficient techniques and those of the other three considered techniques (PPT [16,17], PCT [18,19] and HOS [20]), a first simple comparison can be done, consisting in comparing the profiles along rows of the images. Four rows have been selected, one chosen in the sound zone, with no defect to cross (row \#59), which permits to estimate the background noise level and the other three crossing the whole collection of defects, allowing the assessment of the defect local CNR, edge sharpness and FWHM taken as lateral dimension. All analyzed profiles, for the four techniques, are presented in Figure 7.

\subsubsection{TSR images}

The three graphs of the top row of Figure 7 show that, considering the CNR the sensitivity of the TSR technique to detect defect appears to be less dependent on the defect depth than for the other techniques, at least considering the odd images (5/7 and -7/7). As regard the background noise, the TSR images have a medium noise level, almost identical for the three images. This noise is almost uniform, not influenced by the material structure, which is in favour of a better defect detection, and identical for the three images. As said in Section 4, the TSR coefficient images seem particularly sensitive to the existence of erratic pixels. The profiles in Figure 7 correspond to the images after replacing those pixels by the means of the two immediate neighbours. The impact of this treatment is moderate, changing for instance the initial normalised standard deviation of image TSR $5 / 7$ from $14.6 \%$ to $11.3 \%$.

The sharpness of the two odd images is much better than the one of the even image, as verified for all TSR images [5]. This lower performance was somehow expected since the even image was selected not for its high quality, but because it contains a defect which is barely visible in the odd images (defect \#2-3).

It should be noted that no parameter values were estimated for defects \#3-2, 3-2** and 3-3, since their aspect ratios are too low to be discriminated from noise, and for $\# 3-4^{*}$, which is affected by the overlap with \#1/2-2*.

\subsubsection{PCT images}

The sensitivity to defect appears to rapidly decline with defect depth. The noise increases with the order of the component. In particular the PC4 image is the noisiest of the full collection, with both random and material structure components. Furthermore, the noise is not constant along the line \#59, showing a substantial gradient for the PC4 image. The edge sharpness is comparable to the one of the TSR images.

\subsubsection{PPT images}

The PPT images result from a Fast Fourier Transform (FFT) of the time-evolution of the temperature increase of every pixel. They are characterized by a very good CNR and a very low noise, but suffer from a low sensitivity for deep 
defects. It should be noted that the three selected PPT images were the best ones, as far as defect detection is concerned. Other frequencies were explored but did not enable a better detection of the deepest defects.

\subsubsection{HOS images}

The three HOS images are very similar. Such similarities can be noticed in other publications using the HOS method [20], although this has never been highlighted. They have a fine sensitivity to the shallowest defects, but a poor one for medium and deep defect depths. The noise is comparable to the one of TSR, but more influenced by the material structure and not so uniform across the sample. The similarity between HOS images and the PC2 image can be noticed too.
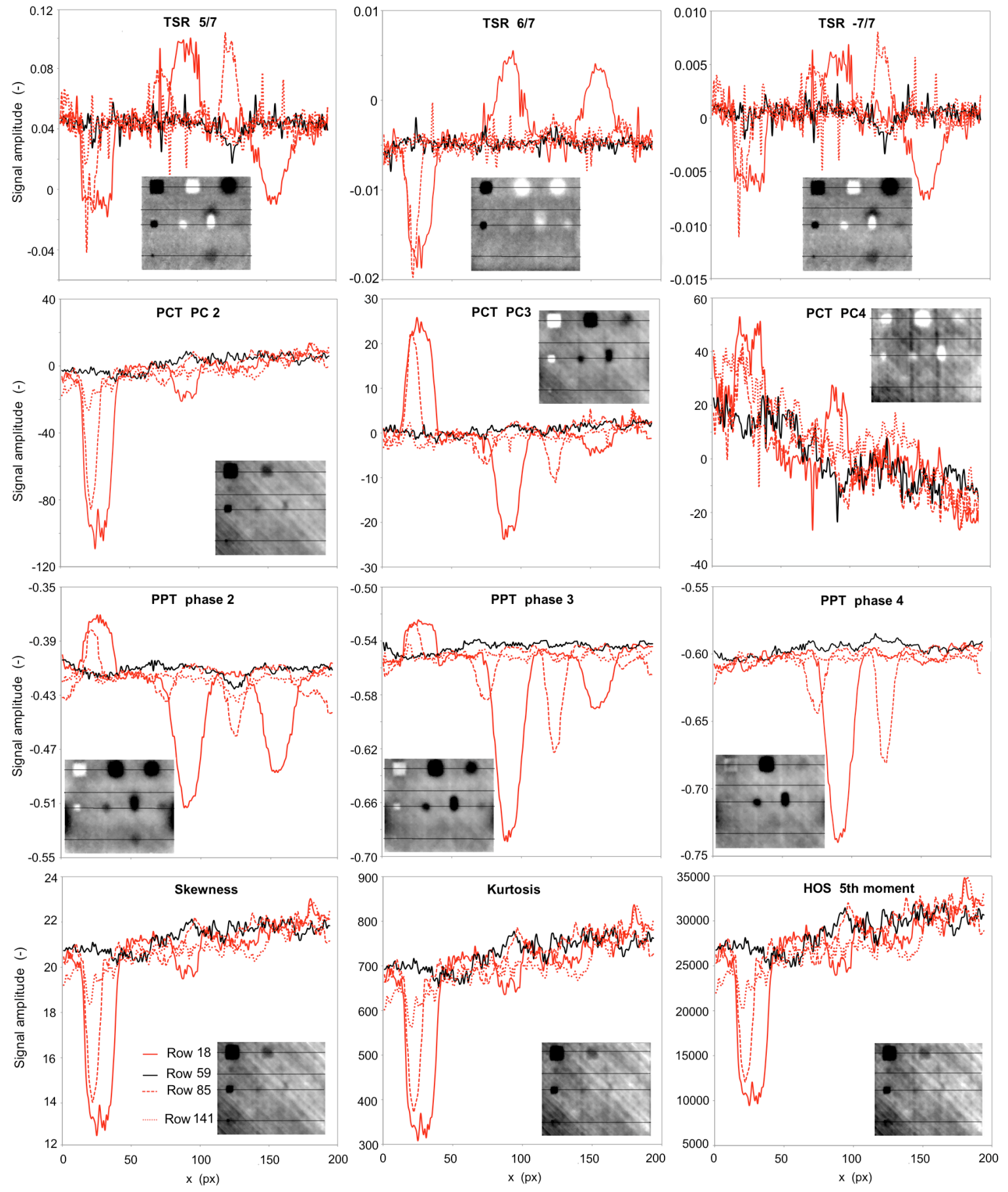

\begin{tabular}{llllll}
\hline Row $18 \quad$ Row $59 \quad$.... & Row 85 & ...... & Row 141 \\
\hline
\end{tabular}

Figure 7. Analysis and comparison of four profiles of the images obtained by the four techniques: TSR, PCT, PPT and HOS. 


\section{Contrast-to-noise ratio (dB)}
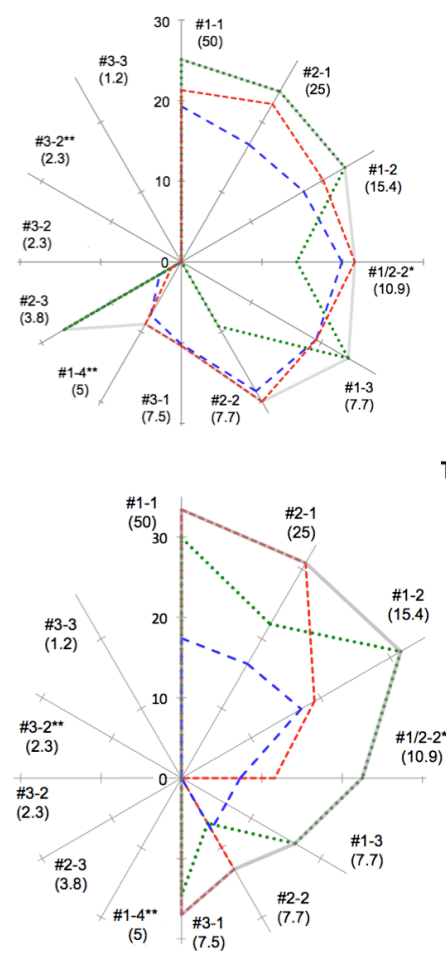

PCT

TSR

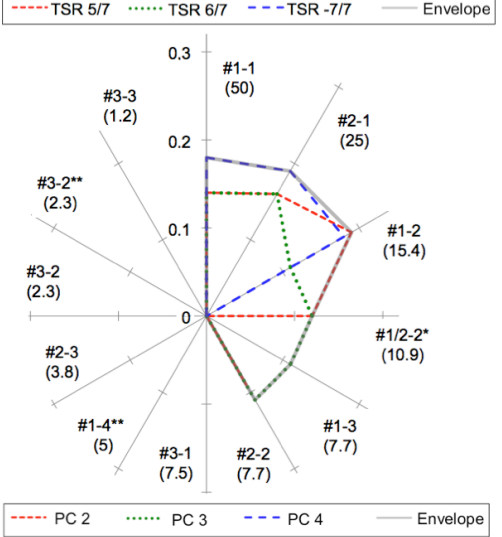

Accuracy on the identified dimension $\left(\log _{10}\left(D_{d} / \Delta D_{d}\right)\right)$
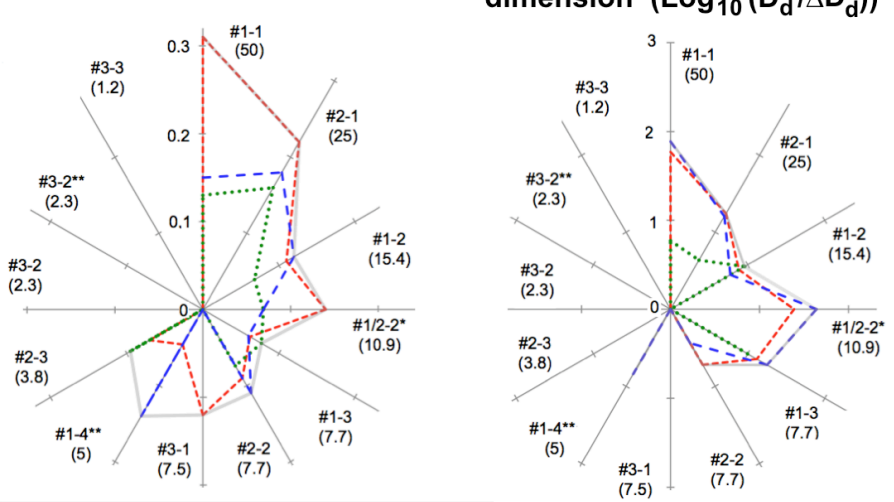
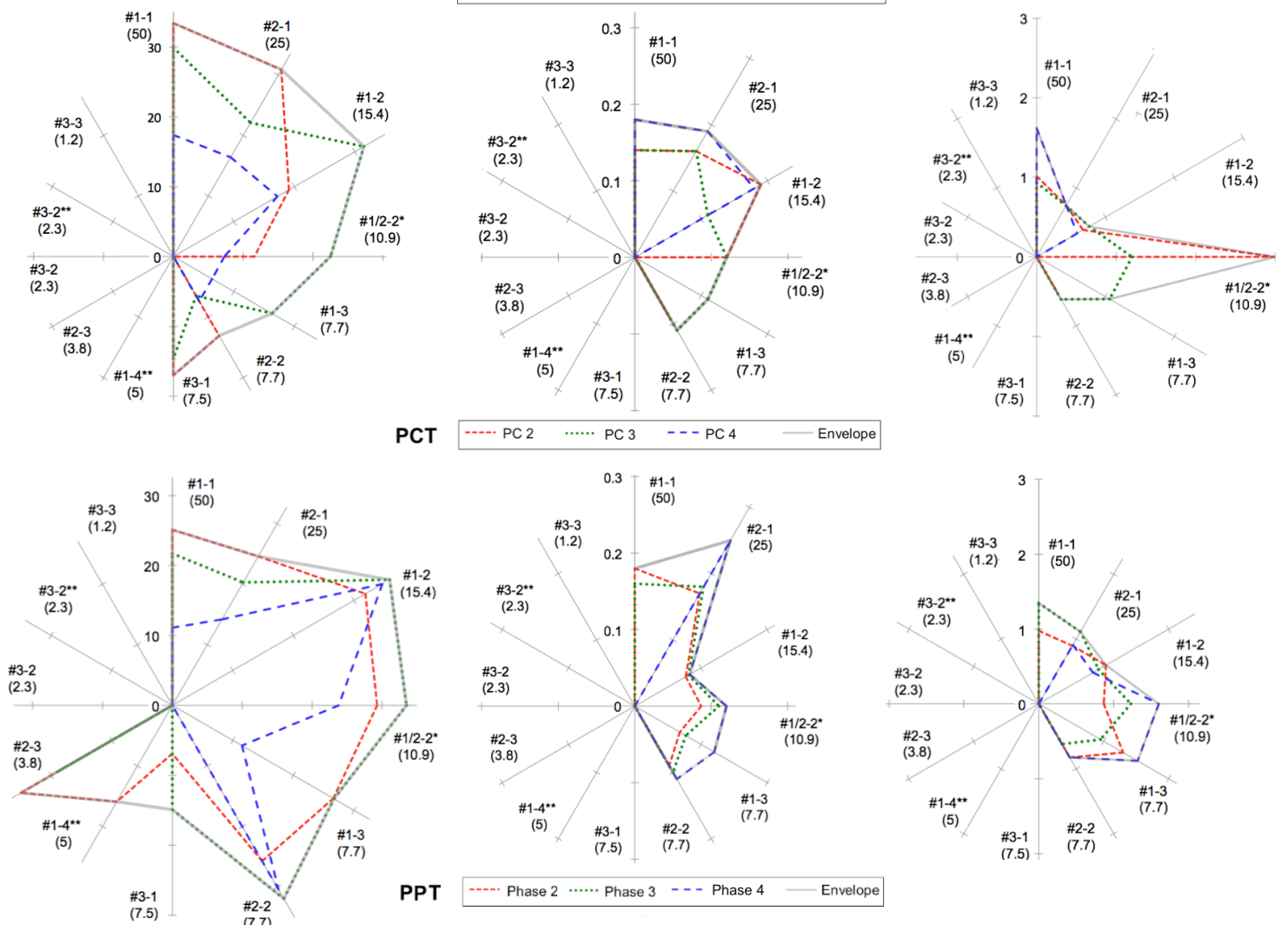

PPT
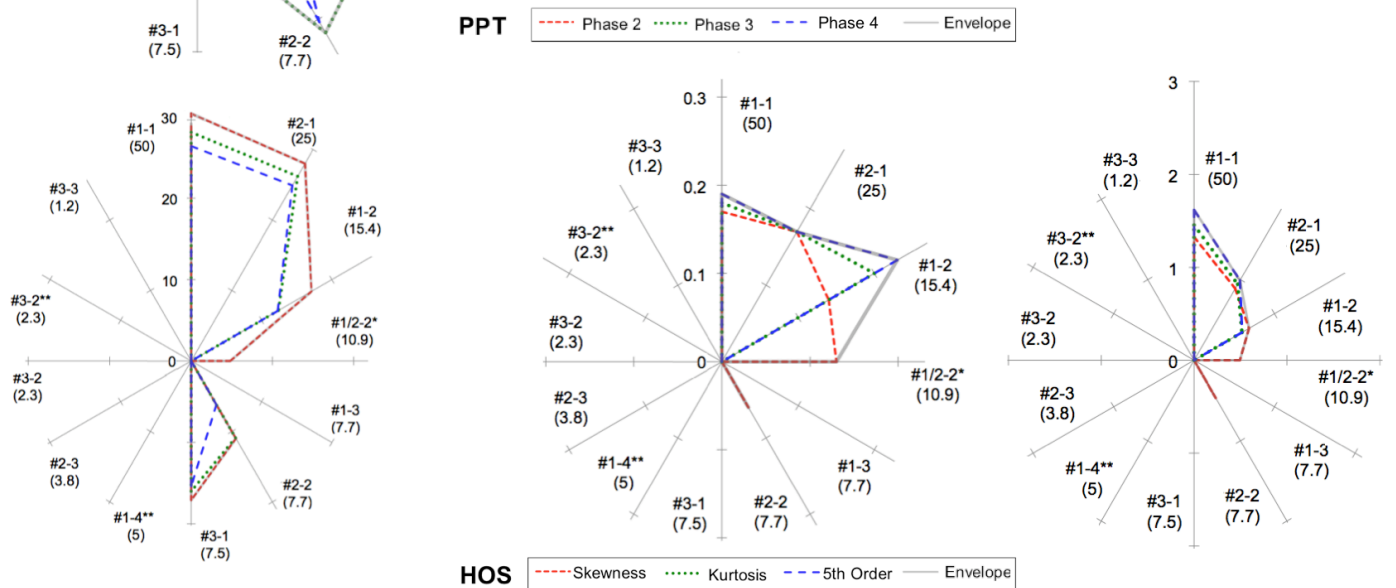

Figure 8. Assessment and comparison of TSR, PCT, PPT and HOS image properties: defect CNR, edge sharpness and accuracy on the identified defect lateral dimension. They are given following decreasing defect aspect ratios. 


\subsection{Quantitative analysis of the quality of the TSR coefficient images and comparison with PCT, PPT and HOS images}

\subsubsection{Compared assessment of the TSR images}

The procedure proposed in the previous section has first been applied to compare the three TSR coefficient images. The results are represented in Figure 8 (top row): for each parameter - CNR, SM, and $\log _{10}\left(\left|D_{d} / \Delta D_{d}\right| F W H M\right)$ - the values resulting from the analysis of the three profiles crossing defects are given, following a clockwise distribution for decreasing aspect ratios (which are given in brackets right next to the defect identification numbers). The envelope of these distributions is also given, representing the best value of the parameter for the trio of images. Radar-type graphs have been chosen to provide the most synthetic presentation and to facilitate the comparisons.

The CNR of the three TSR images is of the same order of magnitude: between 15 and $30 \mathrm{~dB}$ up to aspect ratios from 7 to 8 . Among the three defects \#1-3, 2-2 and 3-1, which have almost the same aspect ratio, only the first two are detected with a CNR of about $20 \mathrm{~dB}$ contrarily to the last one detected with a CNR of $10 \mathrm{~dB}$. This demonstrates that the aspect ratio is not the only governing parameter of defect detection by thermal NDE. For even lower aspect ratios ( 2.3 and $1.2 \mathrm{~dB}$ ), the defects cannot be detected.

The analysis of the sharpness metrics confirms the semi-quantitative analysis given in Section 5.1: the sharpness of the even image is almost lower than the one of the odd images. The influence of the aspect ratio is manifest: when it gets lower, sharpness decreases as well.

The accuracy on the estimation of the defect lateral dimension follows the same trend as the sharpness: odd images lead to better results. The effect of the decrease of the aspect ratio is even more critical, so that it was impossible to properly evaluate the dimensions of defects \#3-1 and 2-3.

\subsubsection{Compared assessment of the four methods.}

Similar results are presented in Figure 8 for the three concurrent methods (rows 2, 3 and 4). In the second row, the PCT results correspond to the second, third and fourth principal component images. The CNRs of the PCT images are slightly higher than the TSR ones for defect aspect ratios larger than 7 but decrease for lower ones (the contrast is drowned in the noise). The sharpness has the same magnitude as the TSR one, but decreases rapidly with the aspect ratio (the contrast plateau no more exists for defects \#3-1, 1-4, 2-3). The accuracy on the defect dimension is very similar to the TSR one, except for defect \#1/2-2* for which a very high accuracy is found, without any simple explanation since the defect signature may be disturbed by the partial overlap of defects existing in this zone.

The PPT results are presented in the third row. The sharpness and accuracy values found for defect dimensions are comparable to the ones of the previous two methods. On the contrary, the CNR reaches values generally higher than those of the other methods, except for the shallowest defects \#1-1 and 2-1. The method appears to be the most efficient one when considering the sole CNR, but presents weaknesses regarding edge sharpness and defect dimension accuracy comparing to the TSR approach.

Finally, the bottom row presents the results of the HOS method. They are generally not so good than those of the other three methods, in particular for deep defects (AR $\leq 10)$, making this method the less performing one.

To get a more synthetic comparison, the same type of representation has been applied to the sole envelopes in Figure 9, allowing a rapid overview of the compared performances of the four methods. The TSR method appears to be not the best one considering the CNR, but obtains the best results for edge sharpness and defect dimension accuracy. This limited performance in CNR is counterbalanced by the fact that, like PPT, it enables to detect deep defects, down to a $4 \mathrm{~mm}$ depth.

Contrast-to-noise ratio $(\mathrm{dB})$

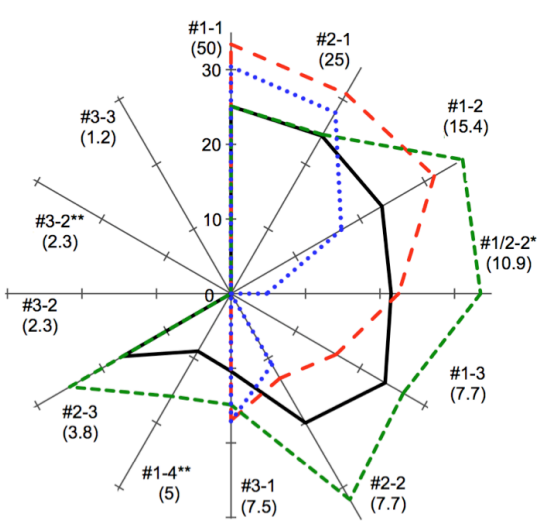

Edge sharpness $\left(\mathrm{pi}^{-1}\right)$

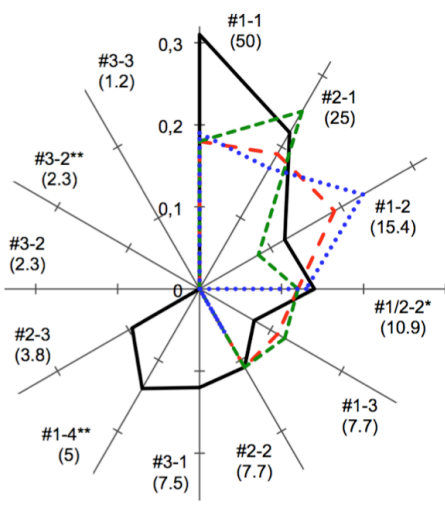

Defect dimension accuracy

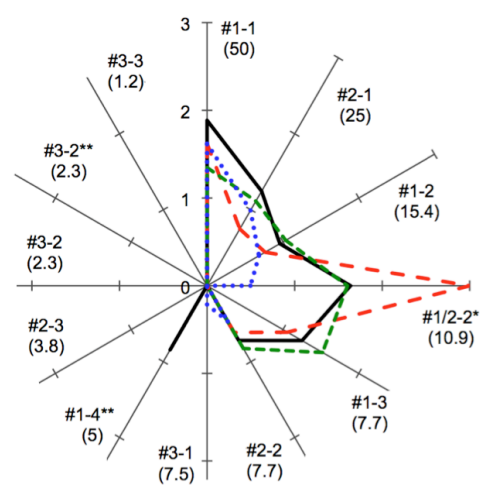

Figure 9. Comparison of TSR, PCT, PPT and HOS image properties: envelopes of the CNR, edge sharpness and accuracy on the identified defect lateral dimension given following decreasing defect aspect ratios. 


\section{Conclusion}

A quantitative assessment of the quality of the defect images produced by the TSR coefficient method and concurrent methods (PCT, PPT and HOS) has been achieved. This assessment is based on the calculation of metrics characterising the defect contrast-to-noise radio and edge sharpness and the accuracy on the defect dimensions when assimilating them to the full-width at half-maximum of the contrast. These metrics have been estimated for a pulse thermography experiment, carried out on a laminate carbon/epoxy coupon containing artificial delamination-like defects, already studied in a previous article aiming at a qualitative assessment of the TSR method [5].

Those three metrics have been assessed from the analysis of row-profiles in the images generated by the four considered methods. These profiles cross both sound and defective regions. The determination of the three metrics has been possible only when the contrast shape presented a plateau pattern. In the other cases only the contrast-to-noise ratio has been evaluated. The resulting graphs illustrate the strong influence of the defect aspect ratio on the method performances and show that other parameters, such as defect depth and material structural noise, have an influence on the defect detection as well.

Regarding the sole contrast-to-noise ratio, the TSR method appears to be less satisfactory than the PPT method, but when it comes to the other two criteria (sharpness and defect dimension characterisation) it turns out to be an efficient tool, even for deep defects, at least as accurate as any other method. This confirms the conclusions drawn from the qualitative study [5].

\section{REFERENCES}

[1] Shepard SM. Advances in pulsed thermography. Thermosense XXIII, Proc. SPIE. 2001;4630:511-515.

[2] Shepard SM, Lhota JR, Rubadeux BA, Wang D, Ahmed T. Reconstruction and enhancement of active thermographic image sequences. Optical Engineering. 2003;42(5):1337-1342.

[3] Balageas D. Defense and illustration of time-resolved pulsed thermography for NDE. Quantitative InfraRed Thermography Journal. 2012;9(1):3-32.

[4] Balageas D, Chapuis B, Deban G, Passilly F. Improvement of the detection of defects by pulse thermography thanks to the TSR approach in the case of a smart composite repair patch. Quantitative InfraRed Thermography Journal. $2010 ; 7(2): 167-187$.

[5] Roche J-M, Leroy F-H, Balageas DL. Images of thermographic signal reconstruction coefficients: a simple way for rapid and efficient detection of dicontinuities. Materials Evaluation. 2014;72(1): 73-82.

[6] Balageas DL, Roche J-M. Common tools for quantitative time-resolved pulse and step-heating thermography - part 1: theroretical basis. Quantitative InfraRed Thermography Journal. 2014;11(1):43-56.

[7] Roche J-M, Balageas DL. Common tools for quantitative pulse and step-heating thermography - Part II: experimental investigation. Accepted for publication in Quantitative InfraRed Thermography Journal. 2015;12(1).

[8] Balageas DL, Déom AA, Boscher DM. Characterization and non destructive testing of carbon-epoxy composites by a pulsed photothermal method. Materials Evaluation. 1987;45(4):461-465.

[9] Krapez J-C, Boscher D, Delpech Ph. Déom A, Gardette G, Balageas D. Time-resolved pulsed stimulated infrared thermography applied to carbon-epoxy non destructive evaluation. Proc. First Conf. on Quantitative Infrared Thermography (QIRT 92), Châtenay-Malabry, France, 1992, pp. 195-200. Available from QIRT Open Archives: http://qirt.gel.ulaval.ca/dynamique/index.php?idD=55, Paper QIRT 1992-029.

[10] Roche J-M, Leroy F-H, Balageas DL. Information condensation in defect detection using TSR coefficients images. XIlth Conference on Quantitative Infrared Thermography, Bordeaux, France, 7-11 July 2014. Available from QIRT Open Archives: www.qirt.org/archives/qirt2014/QIRT2014.htmI\#_Toc391894806, Paper QIRT 2014-002.

[11] Lopez F, Ibarra-Castanedo C. Maldague X, de Paulo Nicolau V. Pulse thermography signal processing techniques based on the 1D solution of the heat equation applied to the inspection of laminated composites. Materials Evaluation. 2014;72(1):91-102.

[12] Usamentiaga R, Venegas P, Guerediaga J, Vega L, López I. A quantitative comparison of stimulation and postprocessing thermographic inspection methods applied to aeronautical carbon fibre reinforced polymer. Quantitative InfraRed Thermography Journal. 2013;10(1):55-73.

[13] Almond DP, Lau SK. Edge effects and a method of defect sizing for transient thermography. Applied Physics Letters. 1993;62(25):3369-71.

[14] Almond DP, Saintey MB, Lau SK. Edge effects and defect sizing by transient thermography. Proc. of QIRT 94 Conference, Sorrento, Italy, 23-26 August 1994. Ed. Europ. Thermique et Industrie, Paris, 1995: pp. $247-252$. Available from QIRT Opens Archives: www.qirt.org/dynamique/index.php?idD=56, Paper \#1994-037.

[15] Balageas DL. In search of early time - an original approach in the thermographic identification of thermophysical properties and defects. Advances in Optical Technologies. 2013: Article ID 314906. Available from: http://dx.doi.org/10.1155/2013/314906.

[16] Maldague X, Marinetti S. Pulse phase infrared thermography. J. Applied Physics. 1996;79:2694-2698.

[17] Ibarra-Castanedo C, Maldague X. Pulsed phase thermography reviewed. Quantitative InfraRed Thermography Journal. 2004;1(1):47-70.

[18] Marinetti S, Grinzato E, Bison PG, Bozzi E, Chimenti M, Pieri G, Salvetti O. Statistical analysis of IR thermographic sequences by PCA. Infrared Physics \& Technology. 2004;46(1-2):85-91.

[19] Rajic N. Principal component thermography for flaw contrast enhancement and flaw depth characterisation in composite structures. Composite Structures. 2002;58(4):521-528.

[20] Madruga FJ, Ibarra-Castanedo C, Conde OM, López-Higuera JM, Maldague X. Infrared thermography processing based on higher-order statistics. NDT\&E International. 2010;43:661-666. 